\title{
The PEP transducer: a new way of measuring respiratory rate in the non-intubated patient
}

\author{
Dennis Dodds, Jonathan Purdy, Chris Moulton
}

\begin{abstract}
Objectives-To explore the use of a pyroelectric polymer (PEP) film as a transducer for a simple respiratory rate monitor and to evaluate the transducer in a laboratory situation.

Methods-Laboratory evaluation of a new pyroelectric transducer for measurement of respiratory rate.

Results-The amplified output from the pyroelectric film produced an excellent respiratory trace when used on a normal spontaneously breathing subject. The transducer is cheap, robust, and reliable. Conclusions-PEP films have the potential to be used as cheap and effective transducers in respiratory rate monitors for non-intubated patients. In the laboratory, they have many desirable characteristics which should now be evaluated in a clinical setting.

( Accid Emerg Med 1999;16:26-28)
\end{abstract}

Keywords: respiratory rate; non-intubated patients; pyroelectric polymer

Respiratory rate is one of the most important physiological parameters. It is a component of most medical and nursing records and is used in many clinical scoring systems. Extremes of respiratory rate indicate the need for urgent intervention. ${ }^{12}$ Moreover, without this parameter, it is impossible to calculate the revised trauma score. This has caused problems for major trauma outcome studies in both the US ${ }^{3}$ and the UK, where, in $1995,60 \%$ of trauma patients did not have their initial respiratory rate recorded (personal communication).

Until the widespread introduction of capnography in the last decade, the measurement of respiration in clinical medicine was dependent on observation alone, although this is known to be highly inaccurate. ${ }^{45}$ The ability to monitor cyclical changes in carbon dioxide concentration with an infrared transducer provided a reliable way of measuring respiratory rate in a patient with an endotracheal tube in situ.

There remains, however, an acknowledged need to monitor respiration in the nonintubated patient. ${ }^{67}$ Capnography has recently been used for this purpose but a nasal cannula is required to allow accurate sampling of exhaled air. ${ }^{8}$ Moreover, the equipment is delicate, expensive, and requires frequent calibration. In the early part of this decade, electrocardiographic (ECG) monitors came onto the market which used changes in transthoracic impedance to detect respiration.
Their apparent simplicity of use-they require only the standard ECG electrodes for both ECG and respiration monitoring-led to calls for their widespread introduction. ${ }^{6910}$ Unfortunately, there are several problems with impedance monitors in clinical usage:

- They are extremely sensitive to patient movements.

- They have slow response times.

- The respiratory tracing is usually of poor quality.

- They are very slow to recognise apnoea.

Many other methods of monitoring respiration have been described but have also been unsuitable for the clinical situation. These have included more sophisticated impedance pneumography, ${ }^{11}$ nasal thermocouples, ${ }^{12}$ acoustic sensors, ${ }^{13-15}$ fibreoptic sensors, ${ }^{16-18}$ extensometers, ${ }^{19}$ and signal processing of photoplethysmographic and other pulse waveforms. ${ }^{2021}$

Ten years ago pyroelectric polymers (PEPs) became readily available. These thin plastic films are self exciting materials which respond to changes in temperature (pyroelectric effect) and also to changes in pressure (piezoelectric effect). As pyroelectric transducers, they were studied by scientists in many disciplines and researchers noted that if a small piece of PEP was incorporated into a face mask it could be used to monitor the respiratory cycle. ${ }^{22}{ }^{23}$ The concept has lain dormant for a decade while the problem of measuring respiration in the non-intubated patient remained unresolved. We have attempted to incorporate a PEP

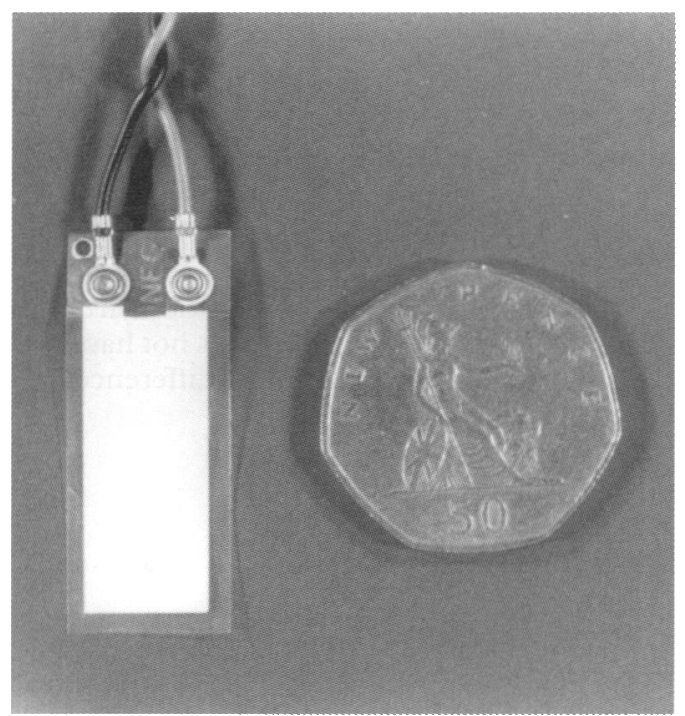

Figure 1 A PEP film transducer $(40 \times 15 \mathrm{~mm})$ alongside an old 50p piece. \\ Department of
Emergency Medicine,
University of
Manchester
C Moulton
Correspondence to:
Dr Chris Moulton, Senior
Lecturer and Honorary
Consultant in Emergency
Medicine, Department of
Accident and Emergency
Medicine, Royal Bolton
Hospital, Minerva Road,
Farnworth, Bolton, Lancs
BL4 0JR.
The authors are members of
the Rembrandt Group, an
academic collaboration
between the Department of
Emergency Medicine of the
University of Manchester
and the Faculty of
Technology of Bolton
Institute. \\ Department of
Emergency Medicine,
University of
Manchester
C Moulton
Correspondence to:
Dr Chris Moulton, Senior
Lecturer and Honorary
Consultant in Emergency
Medicine, Department of
Accident and Emergency
Medicine, Royal Bolton
Hospital, Minerva Road,
Farnworth, Bolton, Lancs
BL4 0JR.
The authors are members of
the Rembrandt Group, an
academic collaboration
between the Department of
Emergency Medicine of the
University of Manchester
and the Faculty of
Technology of Bolton
Institute. \\ Department of
Emergency Medicine,
University of
Manchester
C Moulton
Correspondence to:
Dr Chris Moulton, Senior
Lecturer and Honorary
Consultant in Emergency
Medicine, Department of
Accident and Emergency
Medicine, Royal Bolton
Hospital, Minerva Road,
Farnworth, Bolton, Lancs
BL4 0JR.
The authors are members of
the Rembrandt Group, an
academic collaboration
between the Department of
Emergency Medicine of the
University of Manchester
and the Faculty of
Technology of Bolton
Institute. \\ Department of
Emergency Medicine,
University of
Manchester
C Moulton
Correspondence to:
Dr Chris Moulton, Senior
Lecturer and Honorary
Consultant in Emergency
Medicine, Department of
Accident and Emergency
Medicine, Royal Bolton
Hospital, Minerva Road,
Farnworth, Bolton, Lancs
BL4 0JR.
The authors are members of
the Rembrandt Group, an
academic collaboration
between the Department of
Emergency Medicine of the
University of Manchester
and the Faculty of
Technology of Bolton
Institute. \\ Department of
Emergency Medicine,
University of
Manchester
C Moulton
Correspondence to:
Dr Chris Moulton, Senior
Lecturer and Honorary
Consultant in Emergency
Medicine, Department of
Accident and Emergency
Medicine, Royal Bolton
Hospital, Minerva Road,
Farnworth, Bolton, Lancs
BL4 0JR.
The authors are members of
the Rembrandt Group, an
academic collaboration
between the Department of
Emergency Medicine of the
University of Manchester
and the Faculty of
Technology of Bolton
Institute. \\ Accepted 7 August 1998 \\ Departmen
Electronic
Engineerin \\ Institute \\ J Purdy

Department of
Electronic
Engineering, Bolton
Institute
D Dodds
J Purdy
Department of
Emergency Medicine,
University of
Manchester
C Moulton
Correspondence to:
Dr Chris Moulton, Senior
Lecturer and Honorary
Consultant in Emergency
Medicine, Department of
Accident and Emergency
Medicine, Royal Bolton
Hospital, Minerva Road,
Farnworth, Bolton, Lancs
BL4 OJR.
The authors are members of
the Rembrandt Group, an
academic collaboration
between the Department of
Emergency Medicine of the
University of Manchester
and the Faculty of
Technology of Bolton
Institute.
Accepted 7 August 1998




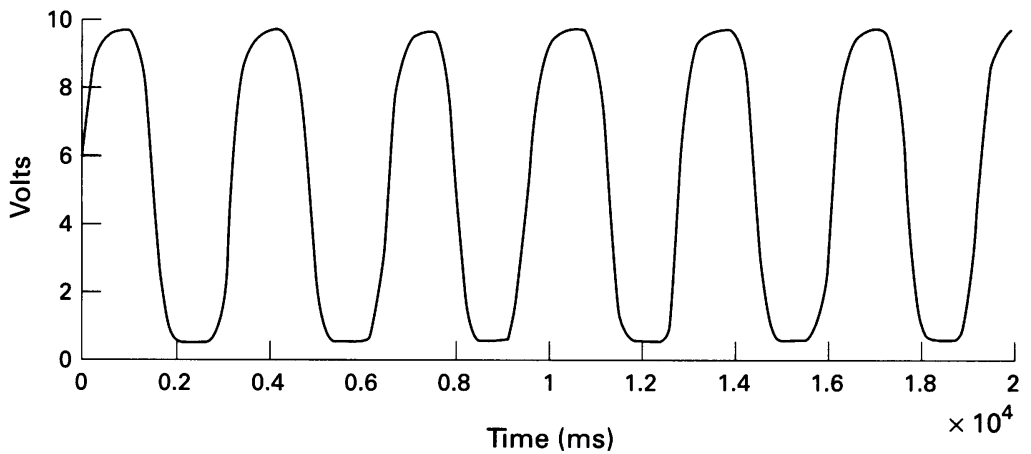

Figure 2 Output from a PEP film transducer (mounted in a standard face mask on a spontaneously breathing subject with a normal respiratory rate).

transducer into a respiratory monitor and demonstrate its efficacy in a laboratory situation.

\section{Laboratory evaluation of a PEP film transducer \\ MATERIALS}

The PEP used was the polarised fluoropolymer, polyvinylidene fluoride (PVDF). This material exhibits a high pyroelectric constant $\left[30 \times 10^{-6} \mathrm{C} /\left(\mathrm{m}^{2} \mathrm{~K}\right)\right]$ and very wide band electronic characteristics ( $\mathrm{DC}$ to $2 \mathrm{GHz}$ ). Its infrared absorption spectrum peaks in the 7-20 $\mu \mathrm{m}$ wavelengths, which corresponds to heat emission from the human body. PVDF is resistant to moisture $(<0.02 \%$ moisture absorption) and most chemicals. It is conformable to complex mouldings and can be fixed in place with standard adhesives.

A small piece of PEP film was found to be adequate as a transducer (fig 1). It was mounted inside a standard oxygen face mask. At this stage, no attempt was made to optimise the transducer design.

\section{SIGNAL PROCESSING}

The temperature differential between inspired and expired gas generates an open circuit output voltage across the transducer of typically $\sim 250 \mathrm{mV}$. To obtain the specimen trace shown in fig 2, a variable gain charge amplifier was used to scale the signal for acquisition by an A-D converter, sampling at a frequency of 2 $\mathrm{kHz}$ and operating under control of a software interface developed by the authors. A simple first order low pass filter was used to band limit the signal to provide a clean trace. The signal

Table 1 Advantages of the PEP film respiratory monitor

- Ideal for the non-intubated patien

- High quality output, unaffected by movement etc

- Fast response time

- Suitable as an apnoea monitor

- Cheap (disposable)

- Easy to use

- Self exciting

- High mechanical strength-damage and impact resistan

Table 2 Comparison of three methods of measuring respiratory rate

\begin{tabular}{lllll}
\hline & $\begin{array}{l}\text { Suitable for } \\
\text { non-intubated } \\
\text { patient }\end{array}$ & Self exciting & $\begin{array}{l}\text { Transient } \\
\text { response }\end{array}$ & Time to show apnoea \\
\hline Transducer type & Yes & Yes & Rapid & One breath \\
Capnograph & No & No & Rapid & One breath \\
Impedance & Yes & No & Slow & $>10$ seconds \\
\hline
\end{tabular}

was converted with 12 bit resolution, level shifted, and scaled to provide amplitude values within a range of $0-10$ volts.

OUTPUT

The amplified output of the system described above (on a subject with a normal respiratory pattern) is shown in fig 2 . The $Y$ axis is in volts (10 volts full scale). The $\mathrm{X}$ axis is labelled in $\mathrm{ms}$ to reflect the sampling rate. Similar traces were obtained with other normal subjects.

\section{Discussion}

The PEP film transducer provides a respiratory tracing which is equal in sensitivity and response times to the output from a capnograph. The film is easily attached to the inside of a standard face mask and, in this nonclinical trial, was not disturbed by the flow of oxygen. As the transducer is self exciting, no power supply is necessary and the wire to the monitor can be led out along the oxygen tubing. Only a small piece of the film is required and this is so cheap $(£ 0.50)$ as to be disposable with its integral mask. (The cost of a typical oxygen mask plus tubing is between $£ 0.97$ and $£ 1.62$.) The transducer can be amplified and configured to provide one channel of a multichannel monitor (perhaps interchangeably with a capnograph) or a second channel on a pulse oximeter.

In the laboratory, the PEP transducer performs well and appears to have many desirable features (table 1). If these advantages are maintained in a clinical setting, PEP based systems will compare favourably with both capnography and transthoracic impedance measurement as a means of monitoring respiration in the nonintubated patient (table 2).

\section{Summary}

Respiratory rate should be measured in all patients who are suffering from: acute illness; major injury; and the effects of sedation or general anaesthesia.

Capnography is the ideal method in those patients who have an endotracheal tube in situ. For patients who are not intubated, the introduction of PEP films has provided the material to make cheap and reliable respiratory transducers for use in face masks-a new answer to a very old problem.

1 Gravelyn T, Weg J. Respiratory rate as an indicator of acute respiratory dysfunction. $\mathcal{F} A M A$ 1980;244:1123-5.

2 McFadden JP, Price RC, Eastwood HD, et al. Raised respiratory rate in elderly patients: a valuable physical sign. $B M \mathcal{F}$ ratory rate in eld

3 Copes WS, Sacco WJ, Champion HR. Editorial. Arch Emerg Med 1989;6:165-8.

4 Krieger B, Feinerman D, Zaron A, et al. Continuous non-invasive monitoring of respiratory rate in critically ill non-invasive monitoring of respira

5 Simoes EA, Roark R, Berman S, et al. Respiratory rate: measurement of variability over time and accuracy at different counting periods. Arch Dis Child 1991;66:1199 203.

6 Clancy MJ, Williams MJ. The respiratory rate. Arch Emerg Med 1991;8:222.

Burdett-Smith $\mathrm{P}$. Always check the respiratory rate. $B M \mathcal{F}$ 1997;314:1549.

8 Egleston CV, Ben Aslam H, Lambert MA. Capnography for monitoring non-intubated spontaneously breathing patients in an emergency room setting. $\mathcal{F}$ Accid Emerg Med 1997;14:222-4.

9 Anderson W, Brock-Utne AJ, Brock-Utne JG, et al. Evaluation of a respiratory rate monitor in post-surgical patients. f Clin Anesth 1992;4:289-91. 
10 Harborne D. Measuring respiratory rate. Arch Emerg Med 1992;9:377-8.

11 Freundlich JJ, Erickson JC. Electrical impedance pneumography for simple non-restrictive continuous monitoring of respiratory rate, rhythm and tidal volume for surgical patients. Chest 1974;65:181-4

12 Marks MK, South M, Carter BG. Measurement of respiratory rate and timing using a nasal thermocouple. $\mathcal{f}$ Clin Monit 1995;11:159-64.

13 Hok B, Wiklund L, Henneberg S. A new respiratory rate monitor: development and initial clinical experience. Int $\mathcal{F}$ Clin Monit Comput 1993;10:101-7.

14 Vegfors $M$, Ugnell $H$, Hok B, et al Experimental evaluation of two new sensors for respiratory rate monitoring. Physio Meas 1993;14:171-81.

15 Sage J, Gough W. A simple inexpensive device for monitoring patient respiration. Med Biol Eng Comput 1998;36:231-2.

16 Lindberg LG, Ugnell H, Oberg PA. Monitoring of respiratory and heart rates using a fibre-optic sensor. Med respiratory and heart rates using
17 Vegfors M, Lindberg LG, Pettersson H, et al. Presentation and evaluation of a new optical sensor for respiratory rate monitoring. Int f Clin Monit Comput 1994;11:151-6.

18 Pettersson $\mathrm{H}$, Stenow EN, Cai $\mathrm{H}$, et al. Optical aspects of a fibre-optic sensor for respiratory rate monitoring. Med Biol Eng Comput 1996;34:448-52.

19 Brimacombe JR, MacFie AG, McCrirrick A. The extensometer-potential applications in anaesthesia and extensometer-potential applications in

20 Nakajima K, Tamura T, Miike H. Monitoring of heart and respiratory rates by photoplethysmography using a digital filtering technique. Med Eng Phys 1996;18:365-72.

21 De Meersman RE, Zion AS, Teitelbaum S, et al. Deriving respiration from pulse wave: a new signal-processing technique. Am $\mathcal{f}$ Physiol 1996;270:H1672-5.

22 Bradley P. Final year BSc degree dissertation. Bolton: Bolton Institute of Higher Education, 1986.

23 Kulkarni V, Cyna A, Hutchison JM, et al. Aura: a new respiratory monitor. Biomed Sci Instrum 1990;26:111-20. 